\title{
Análisis de supervivencia de carcinoma escamoso de laringe en una cohorte retrospectiva chilena del Hospital Carlos Van Buren de Valparaíso
}

\section{Survival analysis of laryngeal squamous cell carcinoma in a Chilean retrospective cohort at the Carlos Van Buren Hospital of Valparaíso}

\author{
Daniel Sotomayor J. ${ }^{1,2}$, Luis Cabezas C. ${ }^{1}$, Francisca Fernández A. ${ }^{1,2}$, \\ Bastián Schuller M. ${ }^{1}$, Rosario Torrejón W. ${ }^{1}$, Cristian Papuzinski A. ${ }^{1,2,3,4}$
}

Departamento de Especialidades, Cátedra de Otorrinolaringología, Escuela de Medicina, Facultad de Medicina, Universidad de Valparaíso. Viña del Mar, Chile. ${ }^{2}$ Servicio de Otorrinolaringología, Hospital Carlos Van Buren. Valparaíso,

Chile

${ }^{3}$ Centro Interdisciplinario de Estudios en Salud (CIESAL), Universidad de Valparaíso. Valparaíso, Chile. ${ }^{4}$ Departamento de Cirugía,

Facultat de Medicina Odontologia, Universitat de València. València, España.

Los autores declaran no tene conflictos de interés.

Recibido el 12 de abril de 2020. Aceptado el 13 de mayo de 2020

Correspondencia: Cristian Papuzinski A. Angamos 655, Edificio R2, Oficina 1107, Reñaca. Viña del Mar, Chile. Email: cristian.papuzinski@uv.cl

\section{Resumen}

Introducción: El cáncer laríngeo es una de las neoplasias de cabeza y cuello más frecuentes, asociado al envejecimiento y a los hábitos de vida. Los análisis de supervivencia de cáncer laríngeo en Chile son escasos. Objetivo: Calcular y analizar la supervivencia y las características clínicas del cáncer laríngeo en pacientes del Servicio de Otorrinolaringología del Hospital Carlos Van Buren, Valparaíso, Chile. Material y Método: Se realizó un estudio retrospectivo de cohorte, incluyendo pacientes diagnosticados con carcinoma escamoso de laringe entre 2007 y 2018. Se calculó la supervivencia con el método de Kaplan-Meier. Se aplicaron las pruebas de log rank, t de Student y exacta de Fisher. Resultados: Se incluyeron 211 pacientes, 90,52\% hombres, con un promedio de edad de 68 años. El factor de riesgo más frecuente fue el tabaquismo. La ubicación tumoral más frecuente fue la glotis $(59,44 \%)$ y el motivo de consulta más común la disfonía (52,66\%). Un 70,48\% presentó estadios avanzados. En 23,92\% se realizó laringectomía total como tratamiento primario. La supervivencia global a 2 años fue de $86,6 \%$ en estadio precoz y $45,2 \%$ en estadio avanzado, mientras que a 5 años fue de $77,4 \%$ y $33 \%$, respectivamente. Conclusión: La supervivencia estimada fue menor que en otros estudios nacionales, lo que puede asociarse al tamaño de la muestra analizada, a factores etarios y/o a mayor latencia de inicio de tratamiento. Se enfatiza el mejoramiento de los registros clínicos y la gestión sanitaria para un manejo oportuno.

Palabras clave: laringe, carcinoma, supervivencia, estudios de cohorte.

\begin{abstract}
Introduction: Laryngeal carcinoma is one of the most frequent head and neck neoplasms, being associated with ageing and lifestyles. In Chile, survival analyses of laryngeal carcinoma are scarce. Aim: To estimate and analyze the survival and clinical characteristics of laryngeal carcinoma in patients attended at the Otorhinolaryngology Department of the Carlos Van Buren Hospital, Valparaíso, Chile. Material and Method: We conducted a retrospective cohort study, including patients diagnosed with laryngeal squamous cell carcinoma between 2007 and 2018. Kaplan-Meier estimator was applied for survival analysis. Log rank test, Student's t-test and Fisher's exact test were applied. Results: 211 patients were included, 90,52\% were men, with an average age of 68 years. The main risk factor was smoking (80\%). The most frequent tumor location was in the glottis $(59,44 \%)$ and the most frequent reason for consultation was dysphonia (52,66\%). Most cases (70,48\%) presented at advanced stages. Laryngectomy was performed as a primary treatment in $23,92 \%$. The 2-year overall survival rate was $86,6 \%$ for early stages and 45,2\% for advanced stages; the 5-year overall survival rate was 77,4\% and 33\%, respectively. Conclusion: The estimated survival rate was lower than those referred by other national studies, which may be associated with the analyzed sample size, age-related factors and/or period of latency until the beginning of treatment. We emphasize the necessity of an improvement in clinical records and the health management to the timely treatment.
\end{abstract}

Keywords: larynx, carcinoma, survival, cohort studies. 


\section{Introducción}

El cáncer de laringe representa el 1,1\% del total de casos de cáncer a nivel mundial al año 2018 ${ }^{1}$. El 90\% corresponde a carcinomas de células escamosas ${ }^{2}$. La tasa de incidencia aumenta con la edad, siendo considerablemente mayor a partir de los 50 años, y predomina en hombres, con una relación de 7:1 respecto a las mujeres ${ }^{1}$. En países como Estados Unidos, cada año se diagnostican aproximadamente 12.000 nuevos casos de cáncer laríngeo, con un resultado de 4.000 muertes al año ${ }^{3}$, mientras que a nivel mundial su incidencia se estima en 4,0 por 100.000 hombres, con una tasa de mortalidad ajustada por edad de 1 por 100.000 habitantes $^{1}$. En Chile, se estima que la incidencia poblacional de cáncer de laringe es de 1,2 casos por cada 100.000 habitantes ${ }^{4-6}$, con una incidencia ajustada por edad de 0,88 por 100.000 personas $^{1}$. Los principales factores de riesgo son consumo de tabaco y alcoholl $^{7-9}$, pero también se han descrito factores genéticos ${ }^{7}$ y la infección por virus papiloma humano ${ }^{10,11}$.

Su ubicación más frecuente es en glotis (65\%), seguido de supraglotis (34\%) y subglotis $(1 \%)^{12}$, pero algunas investigaciones utilizan la clasificación "transglótica" para referirse a carcinomas extensos que cruzan el ventrículo laríngeo y se extienden hacia el espacio paraglótico y la subglotis, donde no es posible definir precisamente su origen ${ }^{13-16}$. Para su etapificación se utiliza la clasificación TNM de la American Joint Committee on Cancer (AJCC), que es específica para cada sitio en la laringe ${ }^{17}$. Sin embargo, hay características comunes para todos los sitios, como por ejemplo, la fijación de una cuerda vocal y la invasión del cartílago tiroides, que corresponden a un T3 y un T4 respectivamente ${ }^{17}$. El cáncer de laringe suele diagnosticarse cuando ya es avanzado, lo que corresponde a los estadios III y IV ${ }^{18}$. Un estudio nacional reportó que, de 83 casos, el 71\% se presentó en estadio avanzado ${ }^{13}$. En cuanto a la terapia, el cáncer de laringe precoz se trata mediante laringectomía transoral parcial y/o radioterapia, mientras que en etapas avanzadas (T4) se realiza laringectomía total que puede asociarse a adyuvancia postoperatoria con radioquimioterapia ${ }^{18}$. De acuerdo con estudios extranjeros, esta patología presenta una supervivencia a cinco años de entre $73 \%$ y $92 \%$ en etapas iniciales y de $50 \%$ a $64 \%$ en etapas tardías ${ }^{19,20}$. En Chile, los estudios de supervivencia con diseño de cohorte para carcinoma escamoso de laringe son muy es$\operatorname{casos}^{13-15,21}$ y no consideran a pacientes de la región de Valparaíso.

\section{Objetivo}

El objetivo de nuestro estudio fue estimar y analizar la supervivencia a cinco años de pacientes con carcinoma escamoso de laringe atendidos en el Hospital Carlos Van Buren de Valparaíso durante 11 años.

\section{Material y Método}

Se realizó un estudio de cohorte retrospectiva que incluyó a todos los pacientes con carcinoma escamoso de laringe atendidos en el Servicio de Otorrinolaringología de Hospital Carlos Van Buren durante los años 2007 a 2018. Este estudio fue aprobado por el Comité Ético-Científico del Servicio de Salud Valparaíso-San Antonio (Acta No 1971). Los participantes fueron incluidos mediante la revisión de registros de egresos hospitalarios. Se revisaron los registros clínicos de cada paciente para obtener los datos de interés. Se obtuvieron los registros de defunción del Servicio de Registro Civil e Identificación Nacional de cada uno de los pacientes. Se excluyeron los portadores de cáncer de laringe con una histología distinta a carcinoma escamoso, los pacientes con cáncer de hipofaringe con compromiso laríngeo y aquellos con registros incompletos que no permitían un análisis apropiado.

Se realizó un análisis de supervivencia mediante curvas de Kaplan-Meier, calculando la supervivencia global desde la fecha del diagnóstico histológico ${ }^{22}$. Para la comparación de curvas se aplicó el test de log rank. Las medias se compararon con el test $\mathrm{t}$ de Student y las proporciones con el test exacto de Fisher. Se consideró un nivel de significancia de 5\%. Los períodos de tiempo se describen con mediana y rango intercuartílico (RIC). Los datos fueron analizados en el programa Stata 16 (Statacorp, Texas, Estados Unidos). 


\section{Resultados}

\section{Características clínicas}

Se incluyeron 211 pacientes, 90,52\% ( $\mathrm{n}=191)$ de sexo masculino, con una razón de 9,55 hombres por cada mujer. La media de edad fue de $68 \pm 10,9$ años, con un rango entre los 34 y los 90 años. Las medias de edad de los pacientes en estadios I-II y III-IV fueron de $68,16 \pm 12,48$ años y 67,9 años $\pm 10,34$ años, sin diferencias significativas intergrupales $(\mathrm{p}=0,88)$. La mayor cantidad de casos $(31,43 \% ; n=66)$ se ubicó en el intervalo etario 61-70 años (Figura 1). Un 79,9\% $(\mathrm{n}=167)$ fue consumidor de tabaco, con una media de 38,1 $\pm 32,6$ paquetes por año, mientras que $58,37 \%$ $(\mathrm{n}=122)$ registró consumo de alcohol.

El tiempo transcurrido entre el inicio de los síntomas y la consulta al especialista fue de 6 meses (RIC 4-12), sin diferencias significativas entre los pacientes diagnosticados en estadio precoz y avanzado. Los motivos de consulta más comunes fueron disfonía $(52,66 \%$; $\mathrm{n}=109)$ y disnea $(28,02 \% ; \mathrm{n}=58)$. La localización anatómica más frecuentemente afectada fue la glotis $(59,44 \% ; n=125)$. La mayor cantidad de pacientes se encontró en estadio AJCC IV $(42,38 \% ; \mathrm{n}=89)$ y con un tamaño tumoral T4 $(35,41 \% ; \mathrm{n}=74)$. La proporción de pacientes con disnea al momento de la consulta tuvo diferencias significativas según tamaño tumoral ( $<<0,001): 1,39 \%$ en T1, $8,33 \%$ en T2, 36, $11 \%$ en T3 y $54,17 \%$ en T4. El 25,84\% $(\mathrm{n}=54)$ presentó metástasis cervicales y el $4,78 \%$ metástasis a distancia al momento del diagnóstico. Se detectó diseminación linfática

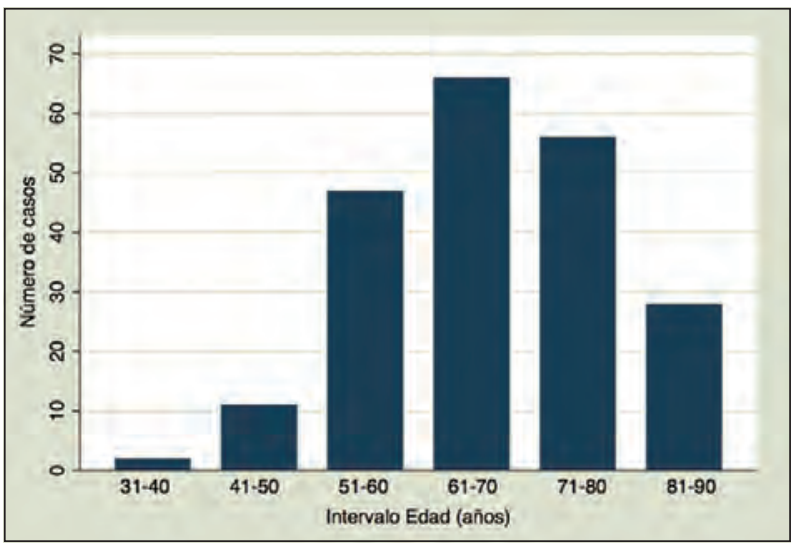

Figura 1. Distribución de casos según intervalo etario. cervical en $15,32 \%(\mathrm{n}=19)$ de los carcinomas glóticos, 47,06\% $(\mathrm{n}=32)$ de los supraglóticos, $14,29 \%(\mathrm{n}=2)$ de los transglóticos y en un paciente con cáncer subglótico. En Tabla 1 se presentan las características de los pacientes incluidos.

\section{Tratamiento}

Entre el diagnóstico histológico del cáncer de laringe y el inicio del tratamiento primario transcurrieron 7,42 semanas (RIC 4,78-12,14), que fue mayor cuando se realizó radioterapia o radioquimioterapia. El tiempo total de tratamiento, definido como la mediana de tiempo entre la fecha de la cirugía y la finalización del manejo adyuvante fue de dieciséis semanas (RIC 14,14-20). La modalidad de tratamiento más común fue la radioterapia exclusiva $31,58 \%(\mathrm{n}=66)$. Se practicó disección cervical en el $66 \%(n=33)$ de los pacientes en quienes se optó por laringectomía total, ya sea disección radical o radical modificada de cuello (la decisión de no realizar disección cervical recayó en el equipo quirúrgico dada la inexistencia de comité oncológico durante parte del periodo estudiado). Se realizó adyuvancia postoperatoria en 35 pacientes. En Tabla 2 se reportan las modalidades de tratamiento de la cohorte.

\section{Supervivencia}

La mediana de supervivencia en los grupos de pacientes en estadio precoz y avanzado fueron de 94,8 y 19,2 semanas, respectivamente. La supervivencia global a 2 años fue de $86,6 \%$ en estadio precoz y de $45,2 \%$ en estadio avanzado, mientras que a 5 años de seguimiento fue de $77,4 \%$ en estadio precoz y de $33 \%$ en estadio avanzado. La supervivencia según estadio precoz o avanzado tuvo una diferencia estadísticamente significativa $(\mathrm{p}<0,001)$ (Figura 2), pero no se observó lo mismo al comparar la supervivencia según sexo $(\mathrm{p}=0,09)$ (Figura 3). De los 211 pacientes, 170 recibieron tratamiento con intención curativa; en este grupo, la supervivencia a 2 y 5 años fue de $89,4 \%$ y $79,7 \%$ en el grupo en estadio precoz, y de $57,6 \%$ y $42,8 \%$ para los pacientes en estadio avanzado, respectivamente, con diferencias estadísticamente significativas $(\mathrm{p}<0,001)$ (Figura 4). Finalmente, se comparó la supervivencia entre los grupos que recibie- 


\section{Tabla 1. Características de los pacientes incluidos}

$\begin{array}{cc}\text { Características } & \text { Total } \\ (\mathrm{n}=211)\end{array}$

Edad al diagnóstico, media \pm DS (años)

$68 \pm 10,9$

Sexo

$191(90,52 \%)$

Masculino, n (\%)

Hábitos

Consumo de alcohol, n (\%)

Consumo de tabaco, n (\%)

$122(58,37 \%)$

Consumo de alcohol y tabaco

$167(79,90 \%)$

Índice de paquete/años, media \pm DS

$106(50,96 \%)$

$38,1 \pm 32,6$

Antecedentes

Cáncer escamoso de cabeza y cuello, n (\%) 2 (0,96\%)

Cáncer de tiroides, n (\%)

$2(0,96 \%)$

Radioterapia cervical, n (\%)

$1(0,48 \%)$

Motivo de consulta

Disfonía, n (\%)

$109(52,66 \%)$

Disfagia, $n(\%)$

$7(3,39 \%)$

Disnea, n (\%)

$58(28,02 \%)$

Disfonía + disfagia y/o disnea, n (\%) $18(3,86 \%)$

Disfonía + masa cervical, n (\%) $\quad 8(3,86 \%)$

Hemoptisis, n (\%)

$3(1,45 \%)$

Disfagia y disnea, $\mathrm{n}(\%)$

$2(0,97 \%)$

$3(1,45 \%)$

Tiempo de evolución de los síntomas

$6(4-12)$

(meses), mediana (RIC)

Sitio anatómico

Glótico, n (\%)

Supraglótico, n (\%)

Transglótico, n (\%)

Subglótico, n (\%)

$125(59,44 \%)$

$68(32,23 \%)$

$14(6,64 \%)$

$4(1,90 \%)$

\section{Estadio AJCC}

I

$38(18,10 \%)$

$24(11,43 \%)$

$59(28,10 \%)$

$89(42,38 \%)$

IV

$39(18,66 \%)$

$27(12,92 \%)$

$69(33,01 \%)$

$74(35,41 \%)$

T4

$155(74,16 \%)$

$14(6,7 \%)$

$34(16,27 \%)$

$6(2,87)$

N3

$199(95,21 \%)$

$10(4,78 \%)$

M1

DS = desviación estándar. RIC = rango intercuartílico
Tabla 2. Modalidades de tratamiento

$\begin{array}{cc}\text { Tratamiento } & \text { Total } \\ (n=209)\end{array}$

Tratamiento primario

Laringectomía total, n (\%)

$50(23,92 \%)$

Laringectomía parcial, n (\%)

$2(0,96 \%)$

Cordectomía, n (\%)

$11(5,26 \%)$

Radioterapia, n (\%)

$66(31,58 \%)$

Radioquimioterapia, n (\%)

$45(21,53 \%)$

Radioterapia paliativa, n (\%)

$10(4,78 \%)$

Otros*, n (\%)

$25(11,96 \%)$

Tratamiento adyuvante $(n=35)$

Radioterapia, n (\%)

$17(48,57 \%)$

Radioquimioterapia, n (\%)

$18(51,42 \%)$

Laringectomía de rescate, $\mathrm{n}(\%)$

11 de 124

(8,87\%)

Tiempos de tratamiento (semanas)

Diagnóstico a cirugía, mediana

4,14

(RIC)

$(2,71-8,14)$

Diagnóstico a RT o RT-QT, mediana

(RIC)

$(6,85-13)$

Cirugía a adyuvancia postoperatoria, mediana (RIC)

9,85

Cirugía a finalización de adyuvancia, mediana (RIC)

$(7,57-14,57)$

16

$(14,14-20)$

$\mathrm{RT}$ = radioterapia. $\mathrm{RT}-\mathrm{QT}=$ radioquimioterapia RIC = rango intercuartílico. * Rechazo de tratamiento, tratamiento no iniciado, solo manejo de dolor.

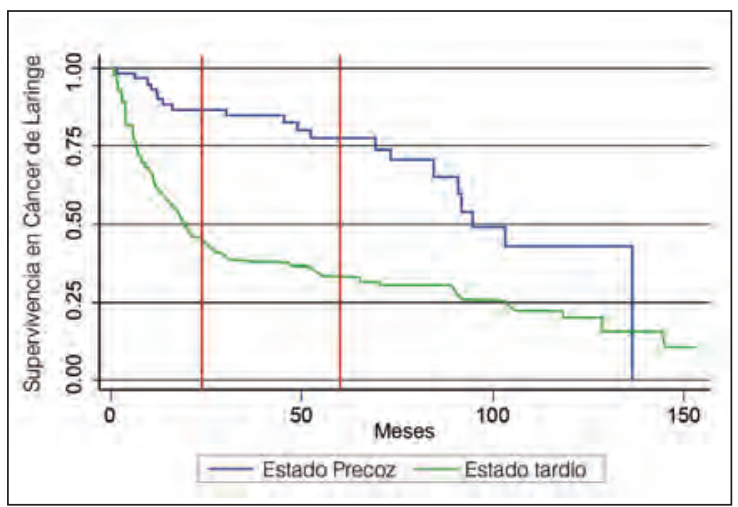

Figura 2. Curvas de supervivencia según estadio. Valor $p<0,001$ (test de log rank). Líneas rojas verticales: seguimiento a 2 y 5 años. 


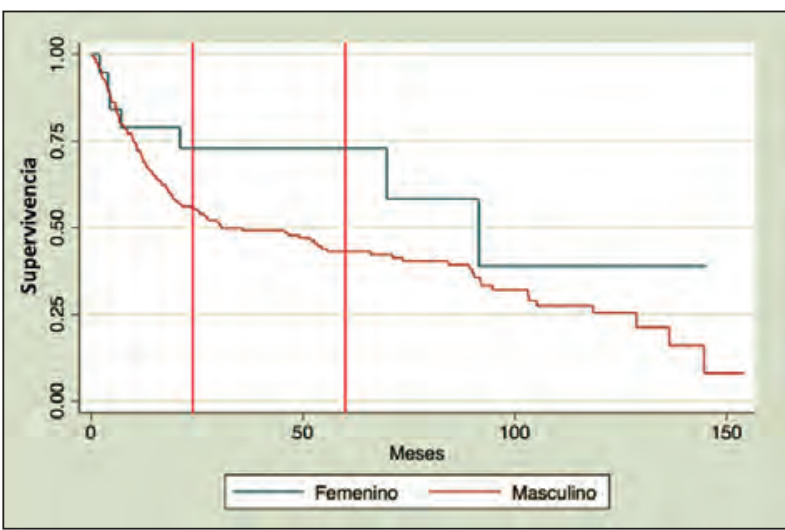

Figura 3. Curvas de supervivencia según sexo. Valor $p=0,09$ (test de log rank). Líneas rojas verticales: seguimiento a 2 y 5 años.

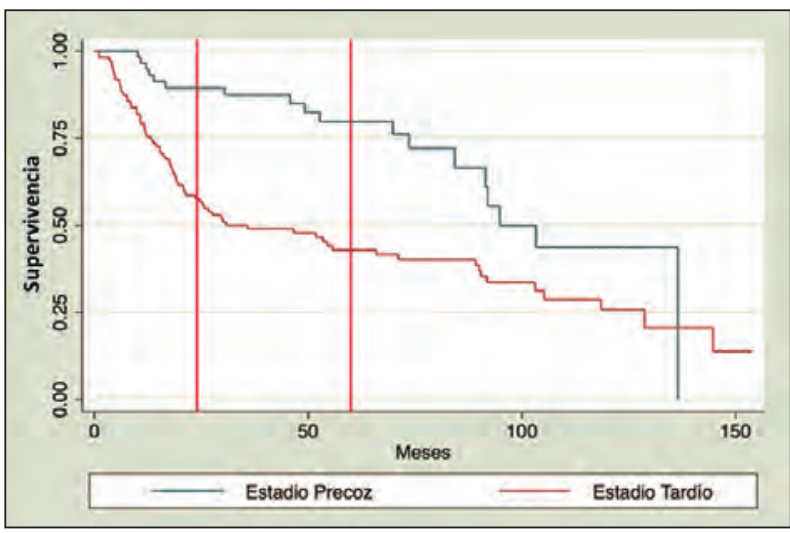

Figura 4. Curvas de supervivencia según estadio en pacientes con intención curativa de tratamiento $(n=170)$. Valor $p<0,001$ (test de log rank). Líneas rojas verticales: seguimiento a 2 y 5 años.

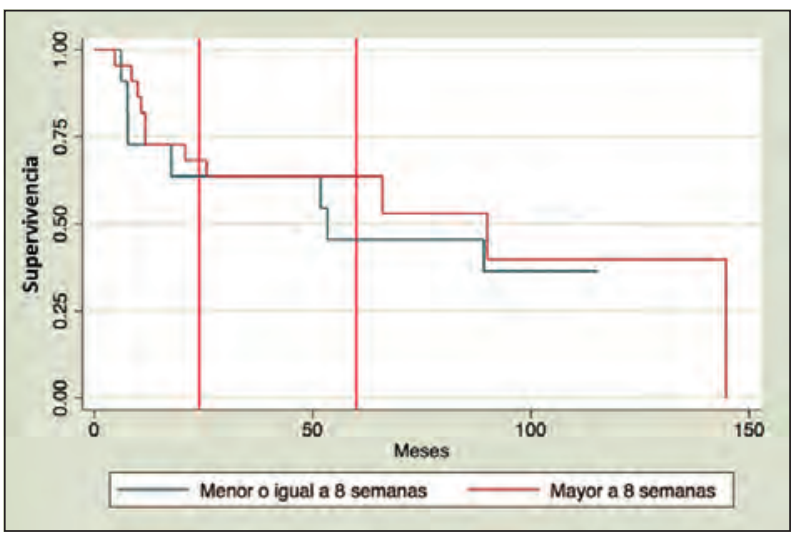

Figura 5. Curvas de supervivencia según tiempo a recibir tratamiento adyuvante postoperatorio. Valor $p=0,2$ (test de log rank). Líneas rojas verticales: seguimiento a 2 y 5 años. ron terapia adyuvante postoperatoria previo o posterior a la semana 8 . No se obtuvieron diferencias estadísticamente significativas entre ambos grupos $(p=0,2)$ (Figura 5). En Tabla 3 se sintetiza el análisis de supervivencia según estadio, sexo, pacientes con intención curativa de tratamiento y pacientes con tratamiento adyuvante.

\section{Discusión}

Este estudio incluyó 211 pacientes con cáncer escamoso de laringe, casi en su totalidad de sexo masculino, con un promedio de 68 años de edad. La media de edad al diagnóstico y la distribución por sexo se asemeja a lo publicado en otras series nacionales ${ }^{13-15,21}$ (Tabla 4). En todos los estudios nacionales, la enfermedad se presenta principalmente en pacientes de sexo masculino. En nuestra cohorte, el promedio de edad (68 años) es ligeramente mayor al estimado en los demás trabajos. Respecto a los factores de riesgo conocidos para el cáncer laríngeo, el más prevalente en nuestro trabajo fue el consumo de tabaco, destacando un elevado consumo promedio de cigarrillos según índice de paquetes por año $(38,1)$; Contreras y cols. ${ }^{15}$ observaron en su centro que el $62,9 \%$ y el $20 \%$ promediaron más de 20 y más de 50 paquetes por año, respectivamente.

Durante la primera consulta, al igual que lo reportado por Pradenas y cols. ${ }^{13}$, la disfonía estuvo presente en más del $90 \%$ de los casos durante la entrevista clínica, sin embargo, la presencia de masa cervical en nuestra muestra fue considerablemente menor (5,3\% versus $32,6 \%)$, lo que puede relacionarse a la inclusión de pacientes con cáncer de hipofaringe en Pradenas y cols. ${ }^{13}$. Si bien encontramos una relación entre tamaño tumoral y la presencia de disnea, no fue posible establecer si este síntoma repercutió en la búsqueda de atención debido a la falta de registro. No obstante, destacamos la variación en la frecuencia de este síntoma, que en tumores pequeños se presentó en 1,39\% de los casos y en cánceres T4 en $54,17 \%$ de los pacientes.

El tiempo que transcurrió entre el inicio de los síntomas y la primera consulta fue de 6 meses (RIC 4-12), una magnitud considerable y mayor que lo informado por otros centros, en donde el promedio alcanza los 5 meses $^{15}$, 


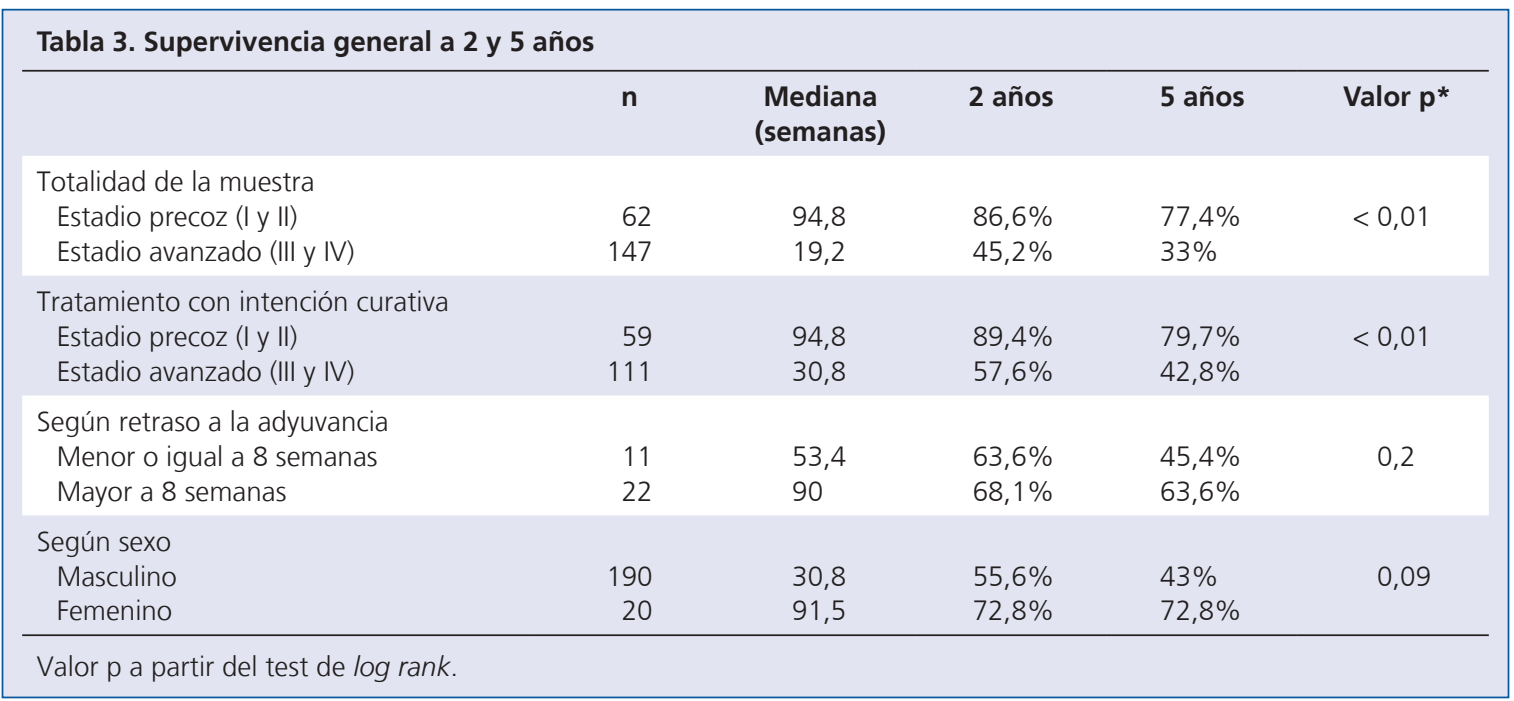

\begin{tabular}{|c|c|c|c|c|c|c|c|c|c|}
\hline Investigación & $\begin{array}{l}\text { Tempo- } \\
\text { ralidad }\end{array}$ & $n$ & $\begin{array}{l}\text { Hombres } \\
\text { (\%) }\end{array}$ & $\begin{array}{l}\text { Promedio } \\
\text { de edad } \\
\text { (años) }\end{array}$ & $\begin{array}{c}\text { Supraglotis } \\
(\%)\end{array}$ & $\begin{array}{l}\text { Glotis } \\
(\%)\end{array}$ & $\begin{array}{l}\text { Subglotis } \\
(\%)\end{array}$ & $\begin{array}{c}\text { Transglótico } \\
\text { (\%) }\end{array}$ & $\begin{array}{l}\text { Estadio } \\
\text { AJCC } \\
\text { Avanzado }\end{array}$ \\
\hline $\begin{array}{l}\text { Hospital Carlos Van Buren } \\
\text { (Sotomayor y cols.) }\end{array}$ & $\begin{array}{l}2007- \\
2018\end{array}$ & 211 & $90,5 \%$ & 68 & $32,2 \%$ & $59,4 \%$ & $1,9 \%$ & $6,6 \%$ & $70,4 \%$ \\
\hline $\begin{array}{l}\text { Hospital Doctor Sótero del } \\
\text { Río (Orellana y cols. 2017) }\end{array}$ & $\begin{array}{l}2005- \\
2011\end{array}$ & 38 & $79 \%$ & 66 & $37 \%$ & $37 \%$ & $0 \%$ & $26 \%$ & $81,5 \%$ \\
\hline $\begin{array}{l}\text { Hospital Barros Luco } \\
\text { Trudeau (Pradenas y cols. } \\
\text { 2004) }\end{array}$ & $\begin{array}{l}1990- \\
2001\end{array}$ & 83 & $93 \%$ & 61,7 & $28,9 \%$ & $32,5 \%$ & $4,8 \%$ & $33,7 \%$ & $71 \%$ \\
\hline $\begin{array}{l}\text { Hospital San Juan de Dios } \\
\text { (Contreras y cols. 2001) }^{15}\end{array}$ & $\begin{array}{l}1994- \\
1999\end{array}$ & 54 & $89 \%$ & 64,3 & $14 \%$ & $48 \%$ & $0 \%$ & $37 \%$ & $74,4 \%$ \\
\hline $\begin{array}{l}\text { Hospital Barros Luco } \\
\text { Trudeau (Contreras y cols. } \\
1989)^{21}\end{array}$ & $\begin{array}{l}1980- \\
1988\end{array}$ & 99 & $87,8 \%$ & 60 & $33,3 \%$ & $45,4 \%$ & $2 \%$ & $19,1 \%$ & $67,6 \%$ \\
\hline
\end{tabular}

debido probablemente a la consulta tardía del paciente y a la lista de espera para la primera consulta. En este sentido, la disfonía es el síntoma que ha presentado la mayor latencia previa al diagnóstico ${ }^{13}$. El retraso en la consulta constituye un problema que pudiese deberse a la evitación a consultar, a un desconocimiento de la población o incluso de los profesionales de atención primaria de salud, en identificar la disfonía crónica como un síntoma que, potencialmente, puede ser causado por un cáncer laríngeo, especialmente en pacientes con factores de riesgo como el consumo de tabaco y alcohol $^{23}$. Un estudio que evaluó los retrasos involucrados en el manejo de pacientes con cáncer de cabeza y cuello, concluyó que uno de los principales factores que retrasa el manejo es la latencia del paciente por consultar, con una mediana de 4,25 meses ${ }^{24}$. No obstante, existen publicaciones que no encontraron asociación entre el tiempo de evolución de los síntomas y el estadio de la enfermedad, lo cual pudiese explicarse por la agresividad intrínseca del tumor ${ }^{25}$.

Respecto a la ubicación tumoral, la principal diferencia con respecto a las otras series se da en los tumores transglóticos, donde observamos una menor frecuencia (Tabla 4). 
Esto puede explicarse porque esta categoría es subutilizada en nuestro centro, dado que al analizar la biopsia se clasifica el tumor según su sitio de origen más probable. Por otra parte, tanto en los reportes nacionales como en esta cohorte, los pacientes fueron mayormente diagnosticados en estadio avanzado de la enfermedad. Concordantemente, las principales modalidades de tratamiento efectuadas fueron la radioterapia o radioquimioterapia en $63,79 \%$ de los 174 pacientes que tuvieron tratamiento con intención curativa, así como laringectomía total en $28,73 \%$. Se reportó una ausencia de tratamiento adyuvante en 30,6\% de los pacientes sometidos a laringectomía total, lo que puede vincularse a la ausencia de un comité oncológico durante los primeros años estudiados en esta investigación. En esta línea, se ha descrito que aproximadamente un $60 \%$ de los pacientes en T3 y T4 que reciben tratamiento quirúrgico deberán ser sometidos a radiación adyuvante ${ }^{26,27}$, empero, esta cifra debe alcanzar el $100 \%$ en los pacientes portadores de un carcinoma T4. Al analizar los tiempos desde el diagnóstico hasta el inicio del tratamiento, se obtuvo una mediana de 4,14 semanas (RIC 2,71-8,14) cuando la cirugía fue el tratamiento primario y de 9,57 semanas (RIC 6,85-13) cuando fue radioterapia o radioquimioterapia. Oberreuter y cols. describen los períodos de tiempo en el manejo de 47 pacientes oncológicos, un $45 \%$ fueron casos de cáncer de laringe; reportan un tiempo similar a nuestro trabajo en aquellos donde el primer tratamiento fue cirugía (mediana de 4 semanas), y un menor período de latencia cuando fue radioquimioterapia (mediana de 8 semanas ${ }^{24}$. Si bien, observamos que en nuestro artículo en ambos grupos se presentaron valores extremos por pacientes que perdieron temporalmente sus controles, el largo tiempo de resolución de los casos quirúrgicos releva la importancia de la gestión en salud, sobre todo cuando la prolongación de estos periodos influye negativamente en el pronóstico de los pacientes ${ }^{24}$. En cuanto al tiempo transcurrido entre la cirugía y el inicio del tratamiento adyuvante, la latencia fue de 9,85 semanas (RIC 7,57-14,57), un lapso prolongado de tiempo, ya que se ha observado que el inicio de la terapia adyuvante posterior a 6 a 8 semanas implica un deterioro del control locorregional y un empeoramiento de la supervivencia ${ }^{16}$. Aunque en nuestro estudio no verificamos una diferencia significativa en las curvas de supervivencia entre los pacientes que recibieron adyuvancia postoperatoria antes y después de 8 semanas (Figura 5), esto puede asociarse al bajo tamaño del subgrupo que recibió adyuvancia (33 pacientes). La prolongación del tiempo al inicio del tratamiento puede obedecer a las características del Servicio de Otorrinolaringología estudiado, ya que corresponde a un Centro que se encarga del tratamiento de todos los casos oncológicos de este tipo en la región de Valparaíso y de gran parte de los casos de la región de Coquimbo.

Como fue exhibido, la supervivencia global de los pacientes en estadios precoces fue de $86,6 \%$ a 2 años y $77,4 \%$ a 5 años, así como $45,2 \%$ a 2 años y $33 \%$ a 5 años para los casos avanzados. Si se obtienen estas mediciones considerando solo los pacientes que fueron tratados bajo una intención de curar la enfermedad, es decir, sin tomar en cuenta pacientes que se mantuvieron en cuidados paliativos, o aquellos que rechazaron el tratamiento, entre otros, se advierte una mayor supervivencia a 2 y 5 años, esencialmente en los pacientes en estadio avanzado (Tabla 3). Un estudio de cohorte $(n=247)$ realizado por Wolf y cols. ${ }^{28}$ (Estados Unidos) estimó una supervivencia general a 5 años de $81 \%$ para estadios I y II y de $72 \%$ para estadios III y IV, superior a la observada en nuestros resultados. De manera más específica, una revisión sistemática que analiza los datos de 24 estudios primarios de pacientes con cáncer de laringe pT4a, reporta una supervivencia general a 2 años de $12 \%$ a $21,2 \%$ cuando fueron tratados con radioterapia, de $30 \%$ a $65 \%$ en el grupo de radioquimioterapia y de $30 \%$ a $100 \%$ cuando se realizó cirugía. A 5 años, los valores variaron de $0 \%$ a $75 \%$ para el grupo de paciente tratados solo con radioterapia, $16 \%$ a $50,4 \%$ para aquellos que recibieron radioquimioterapia y de $10 \%$ a $80,9 \%$ para los tratados quirúrgicamente ${ }^{29}$. En relación con los estudios nacionales, Orellana y cols. ${ }^{14}$ reportaron una supervivencia global en estadio precoz a 2 años y 5 años de $100 \%$ y $71 \%$, respectivamente ( 7 pacientes), mientras que en casos avanzados a 2 y 5 años fue de $56 \%$ y $42 \%$, respectivamente. Por su parte, Pradenas y cols. ${ }^{13}$ informaron una supervivencia en 
casos en estadio III de 71,4\% a 2 años y $42,9 \%$ a 5 años, mientras que en estadio IV fue de un $61,1 \%$ a 2 años y $44,4 \%$ a 5 años. Finalmente, Contreras y cols. ${ }^{15}$, obtuvieron una supervivencia para pacientes con cáncer de laringe en tratamiento con intención curativa de 72,9\%, tanto a 2 como a 5 años para el estadio III, y de $62,9 \%$ y $41,8 \%$ a 2 y 5 años en estadio IV.

Al comparar los hallazgos de estos estudios con nuestros resultados, destaca que presentamos una menor supervivencia a 2 y 5 años en todos los grupos, si consideramos la supervivencia del total de los casos de nuestro trabajo. Por otro lado, al contemplar únicamente los pacientes con intención curativa del tratamiento, observamos porcentajes similares de supervivencia. Sin embargo, la cantidad de casos evaluados por los estudios nacionales es menor que la del presente estudio. Esta investigación presenta un tamaño muestral similar al estudio realizado por Wolf y cols. ${ }^{28}$, no obstante, los resultados no son comparables debido a que los tratamientos estudiados por este grupo difieren de los analizados en el presente estudio. Otro factor que contribuiría a las diferencias entre estudios es la mayor edad de los pacientes incorporados en esta investigación, ya que se ha descrito que, al incluir todas las causas de mortalidad, el grupo de pacientes de 70 o más años presenta una diferencia significativa en su supervivencia ${ }^{31}$. De esta manera, un estudio que incluyó 999 pacientes con cáncer de laringe obtuvo una tasa de supervivencia específica a 5 años de $69 \%$ en pacientes menores de 69 años y de $62 \%$ en mayores de 70 años ${ }^{32}$. Dentro de las limitaciones de este trabajo, una mayor exploración en otros factores clínicos y sociodemográficos que permitieran un mayor análisis de los datos hubiese sido deseable, tales como factores de riesgo cardiovascular, estado nutricional al momento del diagnóstico o nivel socioeconómico. Asimismo, una mejor calidad de los registros hubiese permitido un análisis según causa específica de muerte.

\section{Conclusión}

Esta investigación analizó la supervivencia de una cohorte de pacientes con carcinoma escamoso de laringe, la de mayor tamaño realizada en Chile, aportando hallazgos sobre la realidad nacional y local en este sentido.
Como se ha reportado a nivel nacional e internacional, la predominancia fue masculina, en un rango etario avanzado, el factor de riesgo más frecuente fue el tabaquismo y el motivo de consulta más común la disfonía. La supervivencia fue comparable a estudios internacionales, pero menor que en reportes nacionales, lo que podría explicarse por la diferencia en la cantidad de los pacientes analizados y por la gran demanda asistencial del Centro estudiado, lo que aplazaría los tiempos terapéuticos. Enfatizamos la importancia de la gestión sanitaria para disminuir los tiempos de espera, así como la necesidad de contar con registros clínicos uniformes y estructurados que permitan una adecuada investigación, sobre todo en patologías asociadas a los hábitos de vida y al envejecimiento.

\section{Bibliografía}

1. World Health Organization (WHO). GLOBOCAN, International Agency for Research on Cancer (IARC), 2018.

2. Luers J, Sircar K, Drebber U, Beutner D. The impact of laryngeal dysplasia on the development of laryngeal squamous cell carcinoma. Eur Arch Otorhinolaryngol 2014; 271:539-545. Disponible en https://doi. org/10.1007/s00405-013-2670-2.

3. Kaufman J, Burke A. The etiology and pathogenesis of laryngeal carcinoma. Otolaryngol Clin North Am 1997; 30:1-19.

4. Cardemil F, Ortega G, Cabezas L. Importancia de la epidemiología en cáncer laríngeo: Incidencia y mortalidad por carcinoma escamoso de laringe. Rev Otorrinolaringol Cir Cabeza Cuello 2017; 77:107-112.

5. Ortega G, Cardemil F. Estimación de la incidencia del cáncer de laringe en Chile según la aplicación de un formulario de registro digital. Rev Otorrinolaringol Cir Cabeza Cuello 2017; 77:150-156.

6. MINSAL. Primer informe de registros poblacionales de cáncer de Chile. Quinquenio 2003-2007. In; 2012.

7. Conway DI, Hashibe M, Boffetta P; INHANCE consortium, Wunsch-Filho V, Muscat J, La Vecchia C, Winn DM. Enhancing epidemiologic research on head and neck cancer: INHANCE - The international head and neck cancer epidemiology consortium. Oral Oncol 2009; 45:743-746. Disponible en https://doi. org/10.1016/j.oraloncology.2009.02.007.

8. Purdue MP, Hashibe M, Berthiller J, et al. Type of Alcoholic Beverage and Risk of Head and Neck Cancer - A Pooled Analysis Within the INHANCE Consortium. Am J Epidemiol 2009; 169:132-142. doi: 10.1093/aje/kwn306.

9. Hashibe M, Brennan P, Benhamou S, et al. Alcohol 
Drinking in Never Users of Tobacco, Cigarette Smoking in Never Drinkers, and the Risk of Head and Neck Cancer: Pooled Analysis in the International Head and Neck Cancer Epidemiology Consortium. J Natl Cancer Inst 2007;99:777-789. Disponible en https://doi.org/10.1093/jnci/djk179.

10. IARC Working Group on the Evaluation of Carcinogenic Risks to Humans. Human papillomaviruses. IARC Monogr Eval Carcinog Risks Hum 2007;90:468-471.

11. Mehanna H, Jones T, Gregoire V, Ang K. Oropharyngeal carcinoma related to human papillomavirus. BMJ 2010;340:c1439. Disponible en https://doi.org/10.1136/bmj.c1439.

12. Nocioni R, Molteni G, Mattiuzi C, Lippi G. Updates on larynx cancer epidemiology. Chin J Cancer Res 2020; 32:18-25. Disponible en https://doi. org/10.21147/j.issn.1000-9604.2020.01.03.

13. Pradenas M, Vallejos M, Rahal M, Cisternas A. Cáncer de laringe e hipofaringe. Experiencia del Hospital Barros Luco Trudeau 1990-2001. Rev Otorrinolaringol Cir Cabeza Cuello 2004; 64:13-20.

14. Orellana M, Chuang A, Fulle A, Fernández R, Loyola F, Imarai C. Cáncer de laringe: serie de casos en 6 años en el Complejo Asistencial Doctor Sótero del Río. Rev Otorrinolaringol Cir Cabeza Cuello 2017; 77:35-43.

15. Contreras J, Niklitschek E, Paredes A, Maass J. Cáncer laríngeo-hipofaríngeo. Evaluación en el Hospital San Juan de Dios. Rev Otorrinolaringol Cir Cabeza Cuello 2001; 61:19-30.

16. Contreras P, Muñoz D, Contreras J, Rahal M. Momento de inicio de radioterapia posoperatoria como factor pronóstico en pacientes con cáncer de laringe avanzado. Rev Otorrinolaringol Cir Cabeza Cuello 2018; 78:406-412.

17. Amin M, Edge S, Greene F, et al. AJCC Cancer Staging Manual. 8th ed. New York: Springer International Publishing: 2017.

18. Steuer C, El-Deiry M, Parks J, Higgins K, Saba NF. An Update on Larynx Cancer. CA Cancer J Clin 2017; 67:31-50. Disponible en https://doi.org/10.3322/ caac. 21386 .

19. Nistal C, Bort A, Nocera J, Fiora G. Leucoplasias y su asociación con carcinoma de laringe. Rev FASO 2015; 22:73-78.

20. Papadas T, Alexopoulos E, Mallis A, Jelastopulu E, Mastronikolis N, Goumas P. Survival after laryngectomy: a review of 133 patients with laryngeal carcinoma. Eur Arch Otorhinolaryngol 2010; 267:1095-1101. Disponible en https://doi. org/10.1007/s00405-009-1156-8.

21. Contreras J, Liebner A, Calabrese R, Plandiura J. Cáncer de laringe e hipofaringe: evaluación de los años 1980 a 1988. Rev Otorrinolaringol Cir Cabeza Cuello 1989; 50:25-33.

22. Rich J, Neely J, Paniello R, Voelker C, Nussenbaum B,
Wang E. A practical guide to understanding KaplanMeier curves. Otolaryngol Head Neck Surg 2010; 143:331-336. Disponible en https://doi.org/10.1016/j. otohns.2010.05.007.

23. Tromp D, Brouha X, Hordijk G, Winnubst J, de Leeuw J. Patient factors associated with delay in primary care among patients with head and neck carcinoma: a case-series analysis. Fam Pract 2005; 22:554-559. Disponible en https://doi.org/10.1093/ fampra/cmi058.

24. Oberreuter G, Oliva C, Contreras J, Cardemil F. Análisis de tiempos de espera en pacientes con cáncer de cabeza y cuello en el Hospital San Juan de Dios. Rev Otorrinolaringol Cir Cabeza Cuello 2017; 77:144149.

25. Kaufman S, Grabau J, Loré J. Symptomatology in Head and Neck Cancer: A Quantitative Review of 385 Cases. Am J Public Health 1980; 70:520-522. Disponible en https://doi.org/10.2105/ajph.70.5.520.

26. Skóra T, Nowak-Sadzikowska J, Mucha-Malecka A, Szyszka-Charewicz B, Jakubowicz J, Glinski B. Postoperative irradiation in patients with pT3-4N0 laryngeal cancer: results and prognostic factors. Eur Arch Otorhinolaryngol 2015; 273:673-679. Disponible en https://doi.org/10.1007/s00405-014-3333-7.

27. Graboyes E, Zhan K, Garrett-Mayer E, Lentsch E, Sharma A, Day T. Effect of Postoperative Radiotherapy on Survival for Surgically Managed pT3N0 and pT4aN0 Laryngeal Cancer: Analysis of the National Cancer Data Base. Cancer 2017;123:2248-57. Disponible en https://doi.org/10.1002/cncr.30586.

28. Wolf GT, Bellile E, Eisbruch A, et al. Survival Rates Using Individualized Bioselection Treatment Methods in Patients With Advanced Laryngeal Cancer. JAMA Otolaryngol Head Neck Surg 2017;143:355-366. Disponible en https://doi. org/10.1001/jamaoto.2016.3669.

29. Francis E, Matar N, Khoueir N, Nassif C, Farah C, Haddad A. T4a Laryngeal Cancer Survival: Retrospective Institutional Analysis and Systematic Review. Laryngoscope 2014; 124:1618-1623. Disponible en https://doi.org/10.1002/lary.24557.

30. Pinto J, Nadal A, Mallofré C, et al. El efecto de la edad y de otros parámetros clínico-patológicos en el pronóstico del carcinoma escamoso de laringe: utilidad y limitaciones. Informe preliminar. Rev Otorrinolaringol Cir Cabeza Cuello 2001; 61:95-101.

31. León X, Quer M, Agudelo D, et al. Influence of age on laryngeal carcinoma. Ann Otol Rhinol Laryngol 1998; 107:164-169. Disponible en https://doi. org/10.1177/000348949810700213.

32. Jones A, Beasley N, Houghton D, Husband D. The effects of age on survival and other parameters in squamous cell carcinoma of the oral cavity, pharynx and larynx. Clin Otolaryngol Allied Sci 1998; 23:5156. Disponible en https://doi.org/10.1046/j.13652273.1998.00086.x. 